\title{
Proyecciones
}

Vol. $15, N^{o} 1$, pp. 01 - 17, July 1996

Universidad Católica del Norte

Antofagasta - Chile

\section{EXISTENCE AND MULTIPLICITY OF SOLUTIONS FOR SYSTEMS OF SEMILINEAR ELLIPTIC EQUATIONS*}

\author{
Gastón E. Hernández \\ Universidad Técnica Federico Santa María, Valparaíso, Chile. \\ Yung Sze Choi \\ University of Connecticut, USA.
}

\begin{abstract}
In this work we study the existence and multiplicity of positive solutions of the quasimonotone system of semilinear elliptic equations

$$
\begin{aligned}
& \Delta u_{i}+\lambda f_{i}\left(x, u_{1}, \ldots, u_{m}\right)=0 \quad \text { in } \Omega \\
& \left.u_{i}\right|_{\partial \Omega}=0, i=1, . ., m
\end{aligned}
$$

where $f$ is restricted to a certain type of superlinear growth at infinity.

Key wonds: Elliptic Systems, Quasimonotone.

1980 Mathematics Subject Classification (1985 Revision). Primary 35g. Secondary 35j.
\end{abstract}

"Supported by grant 1941044 of Fondecyt, Chile, and grant 95.12.23 of UTFSM, Chile. 


\section{INTRODUCTION}

There are already a large number of established results [8] on the existence and multiplicity of non-negative solutions for a scalar semilinear elliptic: equation

$$
\begin{gathered}
\Delta u+f(x, u)=0 \\
\left.u\right|_{\partial \Omega}=0
\end{gathered}
$$

in a smooth bounded domain $\Omega$ in $R^{n}, n \geq 2$, where $f(x, 0)>0, f$ is monotone increasing in $u$, and satisfies some increasing rate in $u$ uniformly in $x$. However, as pointed out in [8], the corresponding problem for a system of semilinear elliptic equations is not that well understood. In this work, we shall examime the system of equations

$$
\begin{aligned}
\Delta u_{i}+\lambda f_{i}\left(x, u_{1}, \ldots, u_{m}\right) & =0 \quad \text { in } \Omega \\
\left.u_{i}\right|_{\partial \Omega} & =0, \quad i=1, . ., m
\end{aligned}
$$

where $f_{i}(x, 0, . ., 0)>0$ for all $x \in \Omega, i=1,2, . ., m$. The functions $f_{i}, i=1, \ldots, m$, satisfy the quasimonotone condition and a certain blow up rate as to be made precise in the assumptions (H1) and (H2) below. Then results similar to those of the scalar equation case can be establishred. It should be noted that unless $\frac{\partial f_{i}}{\partial u_{j}}=\frac{\partial f_{j}}{\partial u_{i}}$ for all $1 \leq i, j \leq m$, the problem cannot be formulated in a variational form, hence techniques associated with variational structure are not applicable. The principal tools that we. employ in this work are construction of upper and lower solutions, apriori estimate, and Leray-Schauder degree theory. The proofs are similar to that of [5] where we treat one scalar equation with non-variational structure. In what follows, solution means a classical solution in $\left(\mathcal{C}^{2}(\bar{\Omega})\right)^{m}$.

We will now make our assumptions more precise. For ease of notation, we denote $\vec{u}=\left(u_{1}, \ldots, u_{n}\right)^{T},\|\vec{u}\|_{s}=\left(\sum_{i=1}^{m} u_{1}^{s}\right)^{\frac{1}{s}}$ and $\vec{f}=\left(f_{1}, \ldots f_{m}\right)^{T}$. For equations (3) and (4) defined in a smooth bounded domain $\Omega$ in $R^{n}, n \geq 2$, we assume the following two conditions: 
(H1) For $\vec{x} \in \bar{\Omega}, 1 \leq i, j \leq m, \vec{u} \geq \overrightarrow{0}$ (i.e. $u_{i} \geq 0$ componentwise), let $f_{i} \in \mathcal{C}^{3}\left(\bar{\Omega} \times \mathbb{R}^{m}\right), \frac{\partial \bar{f}_{i}}{\partial u_{i}}(x, \vec{u})>0$ and $\vec{f}$ satisfies the quasimonotone condition $\frac{\partial f_{i}}{\partial u_{j}}(x, \vec{u}) \geq 0$ for $i \neq j$. For sufficiently large $M>0$, there exist constants $c_{1}, c_{2}>0$, independent of $\vec{x}$, such that when $u_{i} \geq M, i=1, \ldots m$, then

$$
\begin{aligned}
f_{i}(\vec{x}, \vec{u}) & \leq c_{2}\|\vec{u}\|_{s}^{s} \\
c_{1} u_{i}^{s} & \leq f_{i}\left(\vec{x}, 0, \ldots, 0, u_{i}, 0, \ldots, 0\right) \text { for all } \vec{x} \in \Omega,
\end{aligned}
$$

where $1<s<\frac{n}{n-2}$ for $n>2$, and any $s \in(1, \infty)$ for $n=2$.

$(H 2) \Omega$ is convex, $\partial \Omega$ has positive curvature everywhere, and there exist $r, \delta>0$ such that for all $\vec{u} \geq 0$ and all $\vec{x} \in \Omega_{r} \equiv\{x \in \Omega \mid \operatorname{dist}(\vec{x}, \partial \Omega)<r\}$, $i=1, \ldots, m$,

$$
\nabla_{x} f_{i}(\cdot, \vec{u}) \cdot \vec{\mu} \leq 0
$$

where $\vec{\mu}$ is a unit vector satifying

$$
|\vec{\mu}-\vec{n}(\vec{x})|<\delta
$$

and $\vec{n}(\vec{x})$ is defined for $\vec{x} \in \Omega$ to be $\vec{n}(\vec{y})$, which is the unit outward normal vector at $\vec{y} \in \partial \Omega$ with $\vec{y}$ defined by $|\vec{y}-\vec{x}|=\operatorname{dist}(\vec{x}, \partial \Omega)$ (the question of well definedness of $\vec{n}(\vec{x})$ is discussed in [4] .)

Under these hypothesis, we shall prove there exists a $\lambda^{*}>0$ such that for $\lambda<\lambda^{*}$, there are at least two solutions, and for $\lambda=\lambda^{*}$, there exists at least one solution, while for $\lambda>\lambda^{*}$, there is no solution. In case that strict convexity of $\vec{f}$ is assumed, i.e., $\left(\frac{\partial^{2} f_{i}}{\partial u_{j} \partial u_{k}}\right)_{j, k=1, \ldots, m}$ is a positive definite matrix for each $i=1, \ldots, m$, then the statements can be made more precise for $\lambda=\lambda^{*}$ : there exists exactly one solution for such $\lambda$, and it is a simple turning point.

Finally we remark that similar techniques can be applied to stucly the existence and multiplicity of the system of quasimonotone semilinear equations

$$
\begin{aligned}
\Delta u_{i}+\vec{b} \cdot \nabla u_{i}+\lambda f_{i}\left(x, u_{1}, \ldots, u_{m}\right) & =0 \\
\left.u_{i}\right|_{\partial \Omega} & =0, i=1, \ldots, m
\end{aligned}
$$

where $\vec{b} \in \mathcal{C}^{1}(\bar{\Omega})$ satifies the additional condition

$$
\vec{b} \cdot \vec{\mu} \geq 0
$$

in assumption ( $\left.\mathrm{H}^{\circ}\right)$. 


\section{Existence of Solutions}

Lor simplicity we use the vectors $\overrightarrow{1}=(1,1, \ldots, 1)$ and $\overrightarrow{0}=(0,0, \ldots, 0)$. When $\lambda=0 . \vec{u} \equiv \overrightarrow{0}$ is the mique sohution. Let $S$ denote the set of nomnegative $\lambda$ for which explations (3)-(1) have a nomnegative solution. We shall begin by proving that $S$ is a bounded interval.

Lemma 2.1. If $\lambda$ is sufficiently small, then $\lambda \in S$.

Proof: Let $\mathbf{n}=\tilde{\phi} \vec{l} \quad$ where

$$
\begin{aligned}
& \Delta \tilde{\phi}=-1, \\
& \left.\tilde{\phi}\right|_{\partial \Omega}=0 \text {. }
\end{aligned}
$$

By the Maxinum Principle, $\tilde{\phi}>0$. Moreover for all $1 \leq i \leq m$, there exists a sufficiently small $\lambda$, independent of $i$, such that

$$
\Delta \tilde{\phi}+\lambda f_{i}(x, \tilde{\phi}, \ldots, \tilde{\phi})=-1+\lambda f_{i}(x, \tilde{\phi}, \ldots, \tilde{\phi})<0,
$$

hence $u_{i}$ is an upper solution of $(1)_{i}$. Consequently $\overrightarrow{\vec{u}}$ is an upper solution for the system of equations (3)-(4). Since $\underline{\vec{u}} \equiv \overrightarrow{0}$ is always a lower solution and the system of equations is quasimonotone, solution exists by monotone iteration methods (Thm. 2 in [9]).

\section{Lemma 2.2.}

If a solution of the equations $(3)-(4)$ exists for $\tilde{\lambda}>0$, then the solution exists for all $\lambda$ such that $0 \leq \lambda \leq \tilde{\lambda}$.

\section{Proof:}

Let $\tilde{u}_{i}$ satisfies (3)-(4) with $\lambda=\tilde{\lambda}$, then $\left(\tilde{u}_{1}, \tilde{u}_{2}, \ldots, \tilde{u}_{m}\right)$ is an upper sohution for

$$
\Delta u_{i}+\lambda f_{i}\left(\vec{x}, u_{1}, \ldots, u_{m}\right)=0, \quad i=1, \ldots, m,
$$

for $0<\lambda<\tilde{\lambda}$. Indeed,

$$
\Delta \tilde{u}_{i}+\lambda f_{i}\left(\vec{x}, \tilde{u}_{1}, \ldots, \tilde{u}_{m}\right)=-\tilde{\lambda} f_{i}\left(\vec{x}, \tilde{u}_{1}, \ldots, \tilde{u}_{m}\right)+\lambda f_{i}\left(\vec{x}, \tilde{u}_{1}, \ldots, \tilde{u}_{m}\right)<0
$$

and $\underline{\vec{u}} \equiv \overrightarrow{0}$ is a lower solution. So, by monotone iteration, there exists a solution $\mathrm{for} 0<\lambda<\tilde{\lambda}$. 
It should be remarked that such monotone iterations give minimal positive solution for each $\lambda$ for which solution exists: There exists a minimal positive solution $\vec{u}$ of (3)-(4) that satisfies $0<\vec{u} \leq \vec{v}$ in $\Omega$ (componentwise) for all positive solution $\vec{v}$ of (3)-(4). It is also clear from the monotone iteration technique that $\vec{u}_{\min }^{\lambda_{1}}<\vec{u}_{\min }^{\lambda_{2}}$ in $\Omega$ for $\lambda_{1}<\lambda_{2}$, where $\vec{u}_{\text {min }}^{\lambda_{i}}$ is the minimal positive solution for $\lambda=\lambda_{j}, j=1,2$.

We will establish there is a second solution besides this minimal positive solution.

Lemma 2.3. $S$ is bounded.

\section{Proof:}

Let $\phi_{1}$ be the eigenfunction corresponding to the smallest eigenvalue $\mu_{1}>0$ of the problem

$$
\begin{aligned}
\Delta \phi+\mu \phi & =0 \\
\phi_{\mid \partial \Omega} & =0 .
\end{aligned}
$$

Now multiply equation (3) by $\phi_{1}$ and integrate over $\Omega$. It follows from the hypothesis in (H1) that we can chowse $\alpha>0$ such that for all $i$ and for all $\vec{x} \in \Omega$

$$
f\left(\vec{x}, u_{1}, \ldots, u_{m}\right) \geq f\left(\vec{x}, 0, \ldots, 0, u_{i}, 0, \ldots, 0\right) \geq \alpha u_{i}
$$

Thus we get

$$
\int_{\Omega} \mu_{1} u_{i} \phi_{1} \mathrm{~d} \vec{x}=\lambda \int_{\Omega} f\left(x, u_{1}, \ldots u_{m}\right) \phi_{1} \mathrm{~d} \vec{x} \geq \lambda \alpha \int_{\Omega} u_{i} \phi_{1} \mathrm{~d} \vec{x} .
$$

Therefore, for any $\lambda \in S$ we have

$$
\lambda \leq \frac{\mu_{1}}{\alpha}<\infty
$$

Hence the lemma.

From the previous lemmas it follows that there exists a $\lambda^{*}=\sup S$ such that a solution exists for $\lambda \in\left[0, \lambda^{*}\right)$ or $\lambda \in\left[0, \lambda^{*}\right]$ and there is no solution for $\lambda>\lambda^{*}$. 


\section{A-priori Bonnds for Solutions}

In this section we will establish that for any solution $\vec{u}$ of $(3)-(4)$ with $\lambda \geq>0$, its $\left({ }^{2}+\alpha-110 \mathrm{rm}\right.$,

$$
\|\vec{u}\|_{C^{2+\alpha}}=\sum_{i=1}^{m}\left\|u_{i}\right\|_{C^{2+\alpha}}
$$

is uniformly bounded in $\Omega$. The bound might depend on $\varepsilon$.

Lemma 3.1. For $\lambda \geq \varepsilon>0$ and any given compact set $K \subset \Omega$ there exists $\tilde{C}>0$, which depends on $K$, such that for all solutions of (3)-(4) (subject to the assiunptions (H1) and (H2)),

$$
\|\vec{u}\|_{L^{1}\left(K^{\prime}\right)}=\sum_{i=1}^{m}\left\|u_{i}\right\|_{L^{1}\left(K^{\prime}\right)} \leq \tilde{C}
$$

\section{Proof:}

For fixed $\lambda$ and $i$, choose $\alpha=\frac{1+\mu_{1}}{\lambda}$. By the superlinearity of $f_{i}$ there is $p>0$, which depends on $\alpha$, such that for all $u_{i}, i=1, \ldots m$, $f_{i}\left(\vec{x}, u_{1}, \ldots, u_{m}\right)>\alpha u_{i}-p$ on $\bar{\Omega}$.

As in equation (6), here we get

$$
\int_{\Omega} \mu_{1} \phi_{1} u_{i} \mathrm{~d} \vec{x}=\lambda \int_{\Omega} f(\vec{x}, \vec{u}) \phi_{1} \mathrm{~d} \vec{x} \geq \lambda \alpha \int_{\Omega} u_{i} \phi_{1} \mathrm{~d} \vec{x}-\lambda p \int_{\Omega} \phi_{1} \mathrm{~d} \vec{x}
$$

Thus by the choice of $\alpha$

$$
\int_{\Omega} u_{i} \phi_{1} \mathrm{~d} \vec{x} \leq \lambda p \int_{\Omega} \phi_{1} \mathrm{~d} \vec{x} .
$$

Since $\lambda \leq \lambda^{*}$ and in any compact set $K \subset \Omega, \phi_{1}$ is bounded below by a constant $c>0$ depending only on $K$, this leads to the uniform boundedness of $\left\|u_{i}\right\|_{L^{1}(K)}$ and so of $\|\vec{u}\|_{L^{1}(K)}$ for any solution $\vec{u}$ of $(3)-(4)$. The value of $\tilde{C}:$ in (1) depends on the compact set $K$.

\section{Lemma 3.2.}

There exists $\delta>0$, that depends only on the geometry of $\Omega$ and $C:>0$ (which can depend on $\delta$, but independent of $\vec{u}$ ) such that if we define

$$
\Omega_{\delta}=\{\vec{x} \in \Omega \mid \operatorname{dist}(\vec{x}, \partial \Omega) \leq \delta\}
$$

then any solution $\vec{u}$ of (3)-(4) has the a-priori bound

$$
\|\vec{u}\|_{L^{\infty}\left(\Omega_{\delta}\right)} \leq C
$$




\section{Proof:}

Wo shall use $r$ and $\delta$ as defined in the assumption (H2). Due to the assumed strict convexity of the domain $\Omega$, if all the $f_{i}$ 's are independent of $\vec{x}$ (xpplicitly. i.e. $f_{i}=f_{i}(\vec{u})$, then we can apply the main theorem in [11] to conclude that any positive solution $\vec{u}$ of the system of equations (3)-(4) is nondecreasing along the inward normal direction for at least some distance $r_{1}<r$ from i) 2 . With the assumption $\nabla_{x} f_{i}(\cdot, \vec{u}) \cdot \vec{n} \leq 0, i=1, \ldots, m$, a similar proof as in $[11]$ can be carried out to yield the same conchusion for the case $\vec{f}=\vec{f}(\vec{r} \cdot \vec{u})$.

Our additional assumptions (H2) on $\vec{f}$ will then allow us to conclude that there exist $0<r_{0} \leq r$ and $0<\delta_{0} \leq \delta$, which depend on the geometry of $\Omega$ but independent of the solution $\vec{u}$, satisfying:

* For any $\vec{x} \in \partial \Omega, u(x-t \vec{\mu})$ is nondecreasing for any $t \in\left[0, r_{0}\right]$, and $\vec{\mu}$ such that $\delta_{0}>|\vec{\imath}-\vec{n}(x)|$.

The proof proceeds exactly as in Step 2 in section 1 of [4]: The statement $\left({ }^{*}\right)$ implies that for $\vec{x} \in \Omega_{\frac{r_{0}}{2}}$, there exists a truncated cone $I_{x}$ as indicated in fig. 1 such that $u(\vec{y}) \geq u(\vec{x})$ for all $\vec{y} \in I_{x}$. Moreover there exists a $\gamma$, independent of $\vec{x} \in \Omega_{\frac{r_{0}}{2}}$, such that $\operatorname{meas}\left(I_{\vec{x}}\right) \geq \gamma>0$ for all $\vec{x} \in \Omega_{\frac{r_{0}}{2}}$. By the lemma 3.2 , for $1 \stackrel{2}{\leq} i \leq m$,

$$
\tilde{C} \geq \int_{I_{\vec{x}}} u_{i}(\vec{y}) \phi_{1}(\vec{y}) \mathrm{d} \vec{y} \geq \gamma u(\vec{x}) \min _{\vec{y} \in I_{\vec{x}}}\left\{\phi_{1}(\vec{y}\} .\right.
$$

Since $\phi_{1}$ is positive in a compact set in $\Omega$ and $r_{0}$ is independent of $\vec{u}$, so the last term on the right hand side has a positive lower bound independent of $\vec{u}$, hence the proof.

From the previous two lemmas since $\vec{u}$ is uniformly bounded near the boundary and locally in $L^{1}$ it follows immediately that

$$
\|\vec{u}\|_{L^{1}} \leq C_{1}
$$

Next we have 
Lemma 3.3. For $\lambda \geq \varepsilon>0$, there exists a $C_{y}>0$ such that for all solutions of (3)-(4),

$$
\|\vec{u}\|_{L^{s}} \leq C_{s}
$$

where $s$ is the blow up rate of $\vec{f}$ as defined in the assumption (H1).

Proof: Multiply equation $(3)_{i}$ by $\tilde{\phi}$ defined in (1)-(2) and integrate to obtain

$$
\begin{aligned}
\int_{\Omega} u_{i} \mathrm{~d} \vec{x} & =\lambda \int_{\Omega} f_{i}\left(\vec{x}, u_{1}, \ldots, u_{m}\right) \tilde{\phi} \mathrm{d} \vec{x} \\
& \geq \lambda \int_{\Omega} f_{i}\left(\vec{x}, 0, \ldots, 0, u_{i}, 0, \ldots, 0\right) \tilde{\phi} \geq \varepsilon c_{0} \int u_{i}^{s} \tilde{\phi}
\end{aligned}
$$

where by our assumptions on $f_{i}$ there exists a sufficiently small $c_{1}>c_{0}>0$ such that $f_{i}\left(\vec{x}, 0, \ldots, 0, u_{i}, 0, . .0\right) \geq c_{0} u_{i}^{s}$. Hence using the result in $(4), u_{i} \in$ $L_{\text {loc }}^{s}$. Together with lemma 3.3 we conclude that there exists a constant $C_{\text {' }}$ such that

$$
\|\vec{u}\|_{L^{s}} \leq C_{s}
$$

for $\lambda \geq \varepsilon>0$.

Lemma 3.4. For $\lambda \geq \varepsilon>0$, there exists a $C>0$ such that for all solutions of (3)-(4)

$$
\|\vec{u}\|_{\mathcal{H}^{1}}=\sum_{i=1}^{m}\left\|u_{i}\right\|_{\mathcal{H}^{1}} \leq C
$$

Proof: From

$$
\Delta u_{i}+\lambda f_{i}\left(\vec{x}, u_{1}, \ldots, u_{n}\right)=0,
$$

we multiply by $u_{i}$ and integrate. By assumption (H1) with $c_{3}=\max \left\{c_{2}, m M^{s} c_{2}\right\}$ we obtain

$$
\begin{aligned}
\int_{\Omega} \frac{1}{2}\left|\nabla u_{i}\right|^{2} \mathrm{~d} \vec{x} & =\lambda \int_{\Omega} f_{i}\left(\vec{x}, u_{1}, \ldots, u_{n}\right) u_{i} \mathrm{~d} \cdot \vec{x} \\
& \leq \lambda^{*} c_{3} \int_{\Omega}\left(\left(u_{1}^{s}+\ldots+u_{n}^{s}\right)+1\right) u_{i} \mathrm{~d} \vec{x}
\end{aligned}
$$


For $i \neq j$ we have

$\int_{\Omega} u_{j}^{s} n_{i} \mathrm{~d} \overrightarrow{\mathrm{r}} \leq\left(\int_{\Omega} u_{j}^{s+1}\right)^{\frac{s}{s+1}}\left(\int_{\Omega} u_{i}^{s+1}\right)^{\frac{3}{s+1}} \mathrm{~d} \vec{r} \leq \frac{s}{s+1}\left\|u_{j}\right\|_{L^{s+1}}^{y+1}+\frac{1}{s+1}\left\|u_{i}\right\|_{L^{s+1}}^{y+1}$

using Hölder's inequality. Adding over all $i$ we arrive at

$$
\sum_{i=1}^{m}\left\|u_{i}\right\|_{i i^{1}} \leq c_{4}\left(\sum_{j=1}^{m}\left\|u_{j}\right\|_{L^{s+1}}^{s+1}+1\right)
$$

for sonter $c_{4}>0$.

From the interpolation inequality

$$
\left\|u_{i}\right\|_{L^{p}} \leq\left\|u_{i}\right\|_{L^{q}}^{\eta}\left\|u_{i}\right\|_{L^{r}}^{I-\eta}
$$

with $\frac{1}{p}=\frac{\eta}{q}+\frac{1-\eta}{r}, 0 \leq \eta \leq 1$, it follows that

$$
\sum_{i=1}^{m}\left\|u_{i}\right\| \mathcal{H}^{1} \leq c_{i}\left(\sum_{i=1}^{m}\left(\left\|u_{i}\right\|_{L^{\prime} n_{c}}^{\eta}\left\|u_{i}\right\|_{L^{s}}^{1-\eta}\right)^{s+1}+1\right)
$$

where $n_{r} \equiv \frac{2 n}{n-2}, \eta=\frac{n_{r}}{(1+s)\left(n_{r}-s\right)}$

Since $\left\|u_{i}\right\|_{L^{s}}$ is bounded we have

$$
\sum_{i=1}^{m}\left\|u_{i}\right\|_{\mathcal{H}^{1}} \leq c_{4}+c_{5} \sum_{i=1}^{m}\left\|u_{i}\right\|_{L^{n_{c}}}^{3}
$$

with $;=n(s+1)<2$ by assumption $(\mathrm{H} 2)$.

I sing Sobolev's estimates for $\left\|u_{i}\right\|_{L^{n_{c}}}$ we arrive at

$$
\|\vec{u}\|_{\mathcal{H}^{1}}^{2} \leq c_{4}+c_{\mathbf{t}}\|\vec{u}\|_{\mathcal{H}^{1}}^{\beta}
$$

IIonce the lenma. 
Lenuma 3.5. For $\lambda \geq \varepsilon>0$, there exists a $6_{0}>0$ such that for all solutions of $(3)-(1)$

$$
\|\vec{u}\|_{C^{2+\alpha}}=\sum_{i=1}^{m}\left\|u_{i}\right\|_{C^{2}} \cdots \leq C_{0}
$$

Proof: Sincer $\left\|u_{i}\right\|_{L^{n_{i}}} \leq\left\|u_{i}\right\|_{\mathcal{H}^{1}} \leq\left(\right.$, the growth restriction on $f_{i}$ innplien; (I'rop. B.1. Appendix B, [10]) that $\left\|f_{i}(\cdot, \vec{u}(\cdot))\right\|_{L^{\gamma}} \leq k_{1}$, with $\gamma>2$.

By the regularity estimate for linear elliptic eculutions [1] for each i. $i=1, \ldots, m,\left\|u_{i}\right\|_{W^{2,2}} \leq K_{2}$. We can now bout-strap, so that the fruction $u_{i}$ is in $C^{2}+x$. Findly, using schauder estinate, $\left\|u_{i}\right\|_{C^{2+a}} \leq r$ for $\lambda \geq \varepsilon>0$.

An immediate consequence of (8) is the existence of solution for $\lambda=\lambda^{*}$.

Lemma 3,6. There exists a minimal positive solntion for $\lambda=\lambda^{*}$. Thus; $S=\left[0, \lambda^{*}\right]$.

\section{Proof:}

Because of lenumb 3.5, we have a $C^{2+\alpha}$ boumd on all solutions. Thus the minimal solution for $\lambda<\lambda^{*}$ converges uniformly in $C(\bar{\Omega})$ to a function $\vec{u}^{*}$ as $\lambda \rightarrow \lambda^{*}$, a standard argment using the regularity of the elliptic equations shows that $\vec{u}^{*}$ is indeed a solution for $\lambda=\lambda^{*}$.

\section{Multiplicity of solutions}

For $0<a<1$, let $T:\left(C^{1+\alpha}(\Omega)\right)^{m} \rightarrow\left(C^{1+\alpha}(\Omega)\right)^{m}$ be the inverse operator of $-\Delta$ subjert to zero Diriclifet boundary conditions. Choose $\delta>0$ sich that $\delta o_{1} \overrightarrow{1}$ is less than the minimal solution at $\lambda=\frac{\varepsilon}{2}$ (recall that $\phi_{1}$ is definerl as in the equations (3)-(4)), and

$$
G=\left\{\vec{u} \in\left(C^{1+\gamma}(\bar{\Omega})\right)^{m} \mid \vec{u} \geq \delta \phi_{1} \overrightarrow{1},\|\vec{u}\|_{c^{1-\alpha}} \leq C_{0}+1\right\} .
$$

Lomma 4.1. Let $\subseteq<\lambda \leq \lambda^{*}<\mu . T h_{h e n} \operatorname{deg}(I-\lambda T(\vec{f}(\vec{x})),,(r, 0)=0$. 


\section{Proof:}

From the previous section we know that uniform a-priori bound can be constructed for $\lambda$ in the range of $\varepsilon \leq \lambda \leq \lambda^{*}<\mu$. As we homotopy from $\varepsilon$ to $\mu$, there cannot be a solution which touches $\delta \phi_{1}$, since the minimal positive solution can only get larger with $\lambda$. Also from the a-priori bounds established in section 3, there is no solution that touches the boundary of the ball $\|\vec{u}\|_{C^{1+\alpha}}=C_{0}+1$, hence the Leray-Schauder degree of the map $I-\lambda T(f((\vec{x}, \cdot))$ for $\varepsilon \leq \lambda \leq \mu$ is well defined.

Since in particular, there is no solution beyond $\lambda^{*}$, we have

$$
0=\operatorname{deg}(I-\mu T(\vec{f}(\vec{x}, \cdot)), G, 0)=\operatorname{deg}(I-\lambda T(\vec{f}(\vec{x}, \cdot)),(r, 0) .
$$

Lemma 4.2. There exists at least 2 solutions of the equation (3)-(4), for $\lambda$ in the range $\left(\varepsilon, \lambda^{*}\right)$.

\section{Proof:}

Let $\lambda \in[\varepsilon, \lambda *)$ be fixed and pick $\lambda_{1}, \lambda_{2}$ such that $\frac{\varepsilon}{2}<\lambda_{1}<\lambda<\lambda_{2}<\lambda^{*}$. Let $\underline{\vec{u}}$ be the minimal positive solution for $\lambda_{1}$ and $\overline{\vec{u}}$ be the minimal positive solution for $\lambda_{2}$. Then $\underline{\vec{u}}$ and $\overline{\vec{u}}$ are lower and upper solutions respectively for $\lambda$. Define

$$
D=\left\{\vec{u} \in\left(C^{1+\alpha}(\bar{\Omega})\right)^{m} \mid \underline{\vec{u}}<\vec{u}<\overline{\vec{u}},\|\vec{u}\|_{C^{1+\alpha}}<C_{0}+1\right\} .
$$

Because the system of equations is quasimonotone, Theorem 1 in [9] gives

$$
\operatorname{deg}(I-\lambda T(\vec{f}(\vec{x}, \cdot)), D, 0)=1 .
$$

Thus by lemma 4.1 and the excision property of the degree we conclude that

$$
\operatorname{deg}(I-\lambda T(\vec{f}(\vec{x}, \cdot)), G \backslash \bar{D}, 0))=-1 .
$$

Hence there exists at least one more solution besides the minimal positive solution for $\varepsilon<\lambda<\lambda^{*}$.

\section{Behavior of the Solution for $\lambda=\lambda^{*}$ for Strictly Convex $\vec{f}$}

In this section we shall further assume that for each $i=1, \ldots, m$, the matrix $\left(\frac{\partial^{2} f_{i}}{\partial u_{j} \partial u_{k}}\right)_{j, k=1, \ldots, m}$ is positive definite and that $\frac{\partial f_{i}}{\partial u_{j}}>0$ for all $1 \leq i, j \leq m$. Then as in the case of a scalar equation we can prove the following properties of the solution set,

(i) The solution for $\lambda=\lambda^{*}$ is unique. 
(ii) Around a neighborhood of $\lambda=\lambda^{*}$, the solution set can be parametrized by $\lambda=\lambda(s)$ and $u=u(s)$ for $-\delta<s<\delta$ for some $\delta>0$, with $\lambda(0)=\lambda^{*}$. Further, $\lambda(s)<\lambda^{*}$ for $s \neq 0$ in that neighborhood. Hence $\lambda=\lambda^{*}$ corresponds to a simple turning point.

To prove these claims, we define

$$
\vec{F}(\vec{x}, \vec{u}, \lambda) \equiv \Delta \vec{u}+\lambda \vec{f}(x, \vec{u})
$$

for $\vec{u}$ in $\left(C^{2+\alpha}(\tilde{\Omega})\right)^{m}$ with zero Dirichlet boundary condition.

Thus solutions of the equations (3)-(4) correspond to

$$
\vec{F}(\vec{x}, \vec{u}, \lambda)=0 .
$$

Denote the minimal positive solution for $\lambda$ by $\vec{u}_{\min }^{\lambda}$. The Frechet derivative of $\vec{F}$ evaluated at the minimal solution is given by

$$
D_{\vec{u}} \vec{F}\left(\vec{x}, \vec{u}_{\min }^{\lambda}, \lambda\right) \vec{v} \equiv \Delta \vec{v}+\lambda D_{\vec{u}} \vec{f}\left(\vec{x}, \vec{u}_{\min }^{\lambda}\right) \vec{v}
$$

for any $\vec{v}$ in $\left(C^{2+\alpha}(\bar{\Omega})\right)^{m}$, where

$$
D_{\vec{u}} \vec{f}\left(\vec{x}, \vec{u}_{\min }^{\lambda}\right)=\left(\frac{\partial f_{i}}{\partial u_{j}}\left(\vec{x}, \vec{u}_{\min }^{\lambda}\right)\right)_{i, j=1, \ldots, m}
$$

First we establish a lemma:

Lemma 5.1. Let $A(\vec{x})=\left(a_{i j}\right)_{i, j=1, \ldots, m}>0$, i.e. $a_{i j}(\vec{x})>0$ for all $1 \leq$ $i, j \leq m$. Then there exists a positive eigenvalue $\eta_{1}$ and a positive vector eigenfunction $\vec{\psi}$ such that

$$
\begin{aligned}
\Delta \vec{\psi}+\eta_{\mathrm{I}} A(\vec{x}) \vec{\psi} & =0, \\
\left.\vec{\psi}\right|_{\partial \Omega} & =0 .
\end{aligned}
$$

Proof: Define $\tilde{T}:(\mathcal{C}(\bar{\Omega}))^{m} \rightarrow(\mathcal{C}(\bar{\Omega}))^{m}$ by $\tilde{T} \vec{\varphi} \equiv-\Delta^{-1}(A \vec{\varphi})$ subjec:t to zero boundary conditions. Then $\tilde{T}$ is a positive operator: If $\vec{\varphi} \geq 0$ with at least one component not identically zero in $\Omega$, then if $\vec{\phi}=\tilde{T} \vec{\varphi}$ we have $-\Delta \vec{\phi}=A \vec{\varphi} \geq \overrightarrow{0}$, with no component being identically zero in $\Omega$. Hence by the Maximum Principle $\vec{\phi}>\overrightarrow{0}$, i.e. each component of $\phi$ is positive on $\Omega$.

Since $\tilde{T}$ is also compact, the Krein-Rutman Theorem implies the existence of a positive eigenvalue $\mu_{1}$ and a positive vector eigenfunction $\vec{\psi}$ such that 


$$
\tilde{T} \vec{\psi}=\mu_{1} \vec{\psi}
$$

so)

$$
A \vec{\psi}=-\mu_{1} \Delta \vec{\psi}
$$

The lemma follows with $\eta_{1}=\frac{1}{\mu_{1}}$.

For a fixed $\lambda_{0}<\lambda^{*}$, by the previous lemma, there exists a positive rigenvalue $\kappa_{1}$ and a positive eigenfunction vector $\vec{\psi}_{1}$ such that

$$
\Delta \vec{\varphi}{ }_{1}+\kappa_{1} \lambda_{0} D_{\vec{u}} \vec{f}\left(\vec{x}, \vec{u}_{\mathrm{min}}^{\lambda_{0}}\right) \varphi_{1}=0 .
$$

hence for $\lambda<\min \left\{\kappa_{1} \lambda_{0}, \lambda_{0}\right\}$ there cannot be a nontrivial solution for the problem

$$
\Delta \phi+\lambda D_{\vec{u}} \vec{f}\left(\vec{x}, \vec{u}_{\min }^{\lambda}\right) \vec{\phi}=0
$$

because of the comparison theorem 1.13 in $[2]$ and $D_{\vec{u}} \vec{f}\left(\vec{x}, \vec{u}_{\min }^{\lambda}\right)$ being an increasing function in $\lambda$ for each entry in the matrix. Thus for sufficiently small $\lambda$ the Frechet derivative in (3) is non-singular. On the other hand there should be a first $\lambda=\bar{\lambda} \leq \lambda^{*}$ at which $D_{\vec{u}} \vec{F}$ becomes singular. Otherwise we can continue the minimal solution branch to $\lambda=\lambda^{*}$ since the solution can never blow up due to the a-priori bound that we have established. I ising Implicit Function theorem at $\lambda=\lambda^{*}$, we can obtain a solution of (2) with $\lambda>\lambda^{*}$, which is a contradiction.

We claim this $\bar{\lambda}$ is the first eigenvalue for

$$
\Delta \psi+\lambda D_{\vec{u}} \vec{f}\left(\vec{x}, \vec{u}_{\min }^{\vec{\lambda}}\right) \vec{\psi}=0
$$

subject to Dirichlet boundary condition, and therefore its corresponding vector eigenfunction $\vec{\psi}$ is positive. If not, there exists a first eigenvalue $\nu_{1}<\dot{\lambda}$ and a corresponding eigenfunction vector $\vec{\psi}_{1}>0$ to the problem

$$
\Delta \overrightarrow{\psi^{\prime}}+\nu D_{\vec{u}} \vec{f}\left(\vec{x}, \vec{u}_{\mathrm{min}}^{\lambda}\right) \vec{\psi} \vec{i}=0 .
$$


However

$$
\nu_{1} D_{u} \vec{f}\left(\vec{x}, \vec{u}_{\text {min }}^{\bar{\lambda}}\right)<\beta D_{\vec{u}} \vec{f}\left(\vec{x}, \vec{u}_{\text {min }}^{\not 3}\right)<\bar{\lambda} D_{\vec{u}} \vec{f}\left(\vec{x}, \vec{u}_{\text {min }}^{\lambda}\right)
$$

for some $3<\lambda$ and close to $\bar{\lambda}$ by simple continuity.

since

$$
\nu_{1} D_{u i} \vec{f}\left(\vec{x}, \vec{u}_{\min }^{\nu_{1}}\right)<\nu_{1} D_{\vec{u}} \vec{f}\left(\vec{x}, \vec{u}_{\min }^{\bar{\lambda}}\right)<\beta D_{\vec{u}} \vec{f}\left(\vec{x}, \vec{u}_{\min }^{3}\right)
$$

thore exist positive first eigenvalues $\rho_{1}, \rho_{2}, \rho_{3}$ to the problems

$$
\begin{aligned}
& \Delta Z_{1}+\rho_{1} \nu_{1} D_{\vec{u}} \vec{f}\left(\vec{x}, \vec{u}_{\text {min }}^{\nu_{1}}\right) Z_{1}=0 \\
& \Delta Z_{2}+\rho_{2} \nu_{1} D_{\vec{u}} \vec{f}\left(\vec{x}, \vec{u}_{\text {min }}^{\bar{\lambda}}\right) Z_{2}=0 \\
& \Delta Z_{3}+\rho_{3} \beta D_{\vec{u}} \vec{f}\left(\vec{x}, \vec{u}_{\text {min }}^{\beta}\right) Z_{3}=0
\end{aligned}
$$

with $\rho_{2}=1$ because of equation $(7)$ and $\rho_{1} \geq \rho_{2} \geq \rho_{3}$ by the comparison theorem 1.13 in [2].

Since $\nu D_{\vec{u}} \vec{f}\left(\vec{x}, \vec{u}_{\mathrm{min}}^{\nu}\right)$ is a continuous function of $\nu$, and eigenvalues depend continuously on the coefficients, hence $\rho$ is a continuous function of $\nu$. By the Intermediate Value Theorem, there exist a $\rho=1$ and a $\nu_{1}<\nu<\beta$ such that

$$
\Delta \vec{Z}+\rho \nu D_{\vec{u}} \vec{f}\left(\vec{x}, \vec{u}_{\min }^{\nu}\right) \vec{Z}=0 .
$$

But this fact contradicts our assumption that $\lambda=\lambda^{*}$ is the first value where the Frechet derivative (3) becomes singular. Hence we have proved that there exists a first eigenvalue $\bar{\lambda} \leq \lambda^{*}$ and a positive vector eigenfunction $\vec{\psi}$ such that

$$
\Delta \vec{\psi}+\vec{\lambda} D_{\vec{u}} \vec{f}\left(\vec{x}, \vec{u}_{\text {min }}^{\bar{\lambda}}\right) \vec{\psi}=0 .
$$

By lemma 5.1, we have the existence of a first eigenvalue $\overline{\bar{\lambda}}$ and a positive vector eigenfunction $\vec{\psi}{ }^{*}>0$ such that

$$
\Delta \overrightarrow{\psi^{*}}+\overline{\bar{\lambda}}\left(D_{\vec{u}} \vec{f}\left(\vec{x}, \vec{u}_{\min }^{\bar{\lambda}}\right)\right)^{T} \vec{\psi}^{*}=0 .
$$

Let $\langle\vec{f}, \vec{g}\rangle=\sum_{i=1}^{m} f_{i} g_{i}$ denote the usual imner product in $\mathbb{R}^{m}$. Take inner product of $(8)$ with $\vec{\psi}^{*}$ and (9) with $\vec{\psi}$. Integrating by parts and substracting, since $\int_{\Omega}\left\langle D_{\vec{u}} \vec{f}, \vec{\psi}\right\rangle \mathrm{d} \vec{x}$ is positive it follows that $\bar{\lambda}=\overline{\bar{\lambda}}$.

Now it can be checked that

$$
\vec{F}_{\lambda}\left(\vec{x}, \vec{u}_{\min }^{\bar{\lambda}}, \bar{\lambda}\right)=\vec{f}\left(\vec{x}, \vec{u}_{\min }^{\bar{\lambda}}\right)>\overrightarrow{0} .
$$


Since $\int_{\Omega}\left\langle\vec{r}^{*} \cdot \vec{f}\left(\vec{x} \cdot \vec{u}_{\min }^{\vec{\lambda}}\right\rangle \mathrm{d} \vec{x}\right.$ is positive, it follows that

$$
\vec{F}_{\lambda} \notin \text { Range }\left(D_{\vec{u}} \vec{f}\left(\vec{x}, \vec{u}_{\min }^{\bar{\lambda}}\right)\right)
$$

So $\lambda=\bar{\lambda}$ is not a bifurcation point [3].

Wo can therefore parametrize the solution set in a neighborhood aromnd $\lambda$ by: $\vec{u}=\vec{u}(s), \lambda=\lambda(s)$, for some sufficient small $\delta>0$ and $-\delta<s<\delta$ with $\lambda(0)=\bar{\lambda}$ as a conserpence of implicit function theorem.

With the assumed smoothmess in $f$, we can differentiate the equation (2) with respect to s, which gives

$$
\Delta \vec{v}+\lambda(s) D_{\vec{u}} \vec{f}\left(\vec{x}, \vec{u}_{\min }^{\lambda}\right) \vec{v}+\lambda^{\prime}(s) \vec{f}\left(\vec{x}, \vec{u}_{\min }^{\lambda}\right)=0
$$

where $\vec{v} \equiv \frac{\mathrm{d} \vec{u}}{\mathrm{~d} s}(s)$. We evaluate the equation at $s=0$, take inner product with $\vec{\psi}^{*}$ and integrate over $\Omega$, which results in

$$
\lambda^{\prime}(0)=0
$$

Differentiate equation (10) once more. With $\vec{w}=\frac{d^{2} \vec{u}}{d s^{2}}(s)$ we have, after evaluating at $s=0$ and using the equation (11),

$$
\Delta w_{i}+\bar{\lambda} \sum_{j=1}^{m} \frac{\partial f_{i}}{\partial u_{j}} w_{j}+\bar{\lambda} \sum_{j, k=1}^{m} \frac{\partial^{2} f_{i}}{\partial u_{j} \partial u_{k}} v_{j} v_{k}+\lambda^{\prime \prime}(0) f_{i}=0
$$

for $i=1, \ldots, m$. Again take inner product with $\vec{\psi}^{*}$ and integrate to get $\lambda^{\prime \prime}(0)<0$ after employing the assumption that $\left(\frac{\partial^{2} f_{i}}{\partial u_{j} \partial u_{k}}\right)_{j, k=1, \ldots, m}$ are positive definite for $i=1, \ldots, m$.

Thus around a neighborhood of $\lambda^{*}$,

$$
\lambda=\bar{\lambda}+\lambda^{\prime \prime}(0) s^{2}+O\left(s^{3}\right) .
$$

So $\bar{\lambda}$ is a simple turning point.

Finally we slow that $\bar{\lambda}=\lambda^{*}$, and there is only one solution for $\lambda=\lambda^{*}$. This will finish the proof of our claims.

Corresponding to $\lambda=\bar{\lambda}$ and $\lambda=\lambda^{*}$ we have

$$
\begin{aligned}
\Delta \vec{u}_{\text {min }}^{\bar{\lambda}}+\bar{\lambda} \vec{f}\left(\vec{x}, \vec{u}_{\text {min }}^{\bar{\lambda}}\right) & =0 \\
\Delta \vec{u}_{\text {min }}^{\lambda^{*}}+\lambda^{*} \vec{f}\left(\vec{x}, \vec{u}_{\text {min }}^{\lambda^{*}}\right) & =0
\end{aligned}
$$

Substract equation (14) from (13), take inner product with $\vec{\gamma}^{*}$ which is the first eigenfunc:tion for $\lambda=\vec{\lambda}$, and integrate to obtain 


$$
\begin{aligned}
\int_{\Omega}\left\langle-\ddot{\lambda} D_{\vec{u}} \vec{f}\left(\vec{x}, \vec{u}_{\min }^{\bar{\lambda}}\right)\left(\vec{u}_{\min }^{\bar{\lambda}}-\vec{u}_{\min }^{\lambda^{*}}\right)\right. & \left.+\bar{\lambda} \vec{f}\left(\vec{x}, \vec{u}_{\min }^{\bar{\lambda}}\right)-\lambda^{*} \vec{f}\left(\vec{x}, \vec{u}_{\min }^{\lambda^{*}}\right), \vec{\psi}^{*}\right\rangle d \vec{x} \\
& =0
\end{aligned}
$$

which can be written as

$$
\begin{aligned}
\bar{\lambda} \int_{\Omega}\left\langle-\vec{f}\left(\vec{x}, \vec{u}_{\text {min }}^{\lambda^{*}}\right)+\vec{f}\left(\vec{x}, \vec{u}_{\text {min }}^{\bar{\lambda}}\right)\right. & \left.+D_{\vec{u}} \vec{f}\left(\vec{x}, \vec{u}_{\min }^{\bar{\lambda}}\right)\left(\vec{u}_{\min }^{\lambda^{*}}-\vec{u}_{\text {min }}^{\bar{\lambda}}\right), \vec{v}^{*}\right\rangle \mathrm{d} \vec{x} \\
& =\int_{\Omega}\left\langle\left(\lambda^{*}-\bar{\lambda}\right) \vec{f}\left(\vec{x}, \vec{u}_{\min }^{\lambda^{*}}\right), \vec{\psi}^{*}\right\rangle \mathrm{d} \mathrm{x}
\end{aligned}
$$

By the convexness assumption on $f$, the left hand side is negative unless $u_{*}=u_{0}$ when it is zero. The right hand side is non-negative since $\lambda^{*} \geq \bar{\lambda}$, and can only be zero when $\lambda^{*}=\bar{\lambda}$. Hence the above equation holds only when $\lambda^{*}=\bar{\lambda}$, and $\vec{u}_{\min }^{\bar{\lambda}}=\vec{u}_{\min }^{\bar{\lambda}}$.

\section{References}

[1] Agmon, S. and Douglis, A. and Nirenberg, L., Estimates near the boundary for solutions of elliptic partial differential equations satifying general boundary conditions, Comm. Pure Appl. Matl., 12 62:3-727, 1959.

[2] H. Amann, Nonlinear operators in ordered Banach Spaces and some applications to nonlinear boundary value problems. Lecture Notes in Mathematics, $543,1-53,1976$.

[3] M.G. Crandall, An introduction to constructive aspects of bifurcation and the implicit function theorem, Application of bifurcation theory, edited by P. Rabinowitz, 1-33, 1977.

[4] D. de Figuereido and P.L.Lions and R.D.Nussbaum, A priori estimates and existence of positive solutions of semilinear elliptic equations, J. Math. Pures et Appl., 61 , 41-63, 1982.

[5] Choi, Y.S. and Hernández, Gastón Existence and Multiplicity of solutions for a Nonvariational Elliptic Problem, Journal of Mathematical Analysis and Its Applications 182 No.1, 189-249, 1994.

[6] Choi, Y.S. and Hernández, Gastón Existence of solutions in a singular biharmonic nonlinear problem, Proceeding of the Edinburgh Math. Soc. $36,537-546,1993$. 
[7] Choi, Y.S. and Hernández, Gastón Behavior of multiple solutions for systems of semilinear elliptic equations Submitted, 1995.

[8] Lions, P.L., On the existence of positive solutions of semilinear elliptic. equations. SIAM Review, 24 No. 4", 441-467, 1982.

[9] McKemna, P.J. and Walter, W., On the Dirichlet Problem for Elliptic: Systems. Applicable Analysis, 21, 207-224, 1986.

[10] P.H. Rabinowitz, Minimax methods in critical point theory with applications to differential equations. C.BMS Regional Conference Series in Mathematics 65, AMS, Providence, 1986.

[11] Shaker, A.W., On Symmetry in Elliptic: Systems, Applicable Analysis, 41, 1-9, 1991.

Received : May 05, 1995

Gastón E. Hernández

Departamento de Matemáticas

Universidad Técnica Federico Santa María

Casilla 110-V

Valparaíso - Chile

y

Yung Sze Choi

Department of Mathematics

Iniversity of Comnecticut

Storr. Comnecticut 06269-3009

Connecticut - U. S. A. 